\title{
Nonlinear dynamics of a cold collisional electron plasma
}

G. Brodin, and L. Stenflo

Citation: Physics of Plasmas 24, 124505 (2017);

View online: https://doi.org/10.1063/1.5011299

View Table of Contents: http://aip.scitation.org/toc/php/24/12

Published by the American Institute of Physics

\section{Articles you may be interested in}

Analytical electron acoustic solitary wave solution for the forced $\mathrm{KdV}$ equation in superthermal plasmas

Physics of Plasmas 24, 122106 (2017); 10.1063/1.4994562

Effect of collisions on Weibel instability with anisotropic electron distributions

Physics of Plasmas 24, 122113 (2017); 10.1063/1.4990111

Self-organized criticality in a cold plasma

Physics of Plasmas 24, 120701 (2017); 10.1063/1.5005560

Nonlinear waves and coherent structures in quasi-neutral plasmas excited by external electromagnetic radiation Physics of Plasmas 24, 123105 (2017); 10.1063/1.5003322

Particle-in-cell simulation of Buneman instability beyond quasilinear saturation

Physics of Plasmas 24, 122103 (2017); 10.1063/1.5006463

Solitary structures in an inhomogeneous plasma with pseudo-potential approach

Physics of Plasmas 24, 114502 (2017); 10.1063/1.4993812

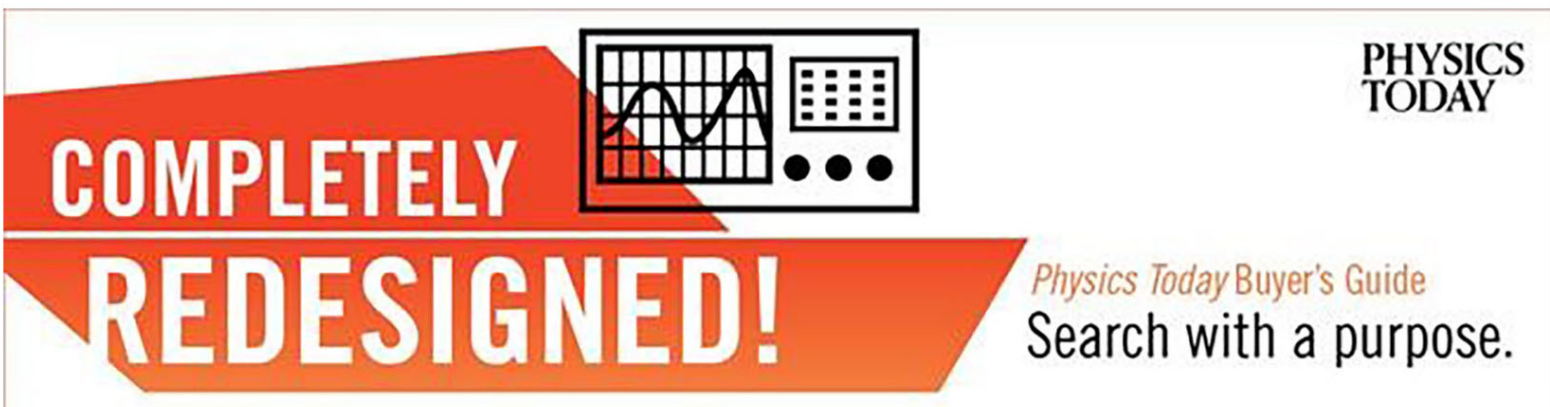




\title{
Nonlinear dynamics of a cold collisional electron plasma
}

\author{
G. Brodin ${ }^{1}$ and L. Stenflo ${ }^{2}$ \\ ${ }^{1}$ Department of Physics, Umea University, SE-901 87 Umeå, Sweden \\ ${ }^{2}$ Department of Physics, Linköping University, SE-581 83 Linköping, Sweden
}

(Received 31 October 2017; accepted 5 December 2017; published online 22 December 2017)

We study the influence of collisions on the dynamics of a cold non-relativistic plasma. It is shown that even a comparatively small collision frequency can significantly change the large amplitude wave solution. Published by AIP Publishing. https://doi.org/10.1063/1.5011299

The theory of weakly nonlinear plasma waves has been developed during more than half a century (e.g., Refs. 1-3). Nowadays, increasing interest is therefore instead paid to strongly nonlinear plasma waves (e.g., Refs. 4-14). In order to avoid too many mathematical difficulties, one then has to focus on very simple and basic plasma phenomena. One typical example concerns the simple large amplitude solutions for electron plasma waves. In the present brief communication, we shall thus reconsider this case in order to illustrate the very significant effects of collisions on the singularities which appear in the collisionless case.

The electrostatic oscillations in a cold one-component (electron) plasma are governed by the electron continuity and momentum equations together with the Poisson equation. Considering the simple one-dimensional case with oscillations along the $x$-axis, we write these basic equations in the form

$$
\begin{gathered}
\partial_{t} n+\partial_{x} n v=0, \\
\partial_{t} v+v \partial_{x} v=\frac{q_{e}}{m_{e}} E-\nu v,
\end{gathered}
$$

and

$$
\partial_{x} E=\frac{q_{e}}{\varepsilon_{0}}\left(n-n_{0}\right),
$$

where $n$ is the electron density, $v$ is the electron fluid velocity, $n_{0}$ is the density of the heavy (here immobile) ions, $q_{e} / m_{e}$ is the electron charge to mass ratio, and $\nu$ is the electron collision frequency. We can here easily eliminate the electric field by applying $\partial_{x}$ on both sides of the momentum equation. What then remains is the electron continuity equation (1) together with the electron velocity equation

$$
\partial_{x}\left[\partial_{t} v+v \partial_{x} v+\nu v\right]=\omega_{p}^{2} \frac{\left(n-n_{0}\right)}{n_{0}},
$$

where $\omega_{p}=\left(n_{0} q_{e}^{2} / \varepsilon_{0} m_{e}\right)^{1 / 2}$.

Instead of the variables $n$ and $v$, we now choose to use the two dimensionless variables $N(x, t)=n / n_{0}$ and $V(x, t)$ $=\partial_{x} v / \omega_{p}$, which are governed by the two coupled equations (1) and (4).

Let us next first reconsider the collisionless case $(\nu=0)$ and look for the simple solutions where both $N$ and $V$ are only functions of time. Using Eq. (1) to eliminate $V$ in Eq. (4), we then obtain the simple, and EXACT, solution ${ }^{12}$

$$
N(t)=\frac{1}{1+\Delta \cos \left(\omega_{p} t\right)}
$$

and

$$
V(t)=-\frac{\Delta \sin \left(\omega_{p} t\right)}{1+\Delta \cos \left(\omega_{p} t\right)},
$$

where $\Delta$ is a constant amplitude. An arbitrary phase factor $\varphi$ can, of course, be added to the solution, i.e., $\omega_{p} t \rightarrow \omega_{p} t+\varphi$, but this is not needed here.

The solutions (5) and (6) are obviously not valid if $\Delta \geq 1$, as $n$ cannot be infinitely large. In Ref. 5 , the authors kept a finite collision frequency $\nu$ in the momentum equation, but assumed that $\nu$ was constant. This modified the solutions to some extent, although the singularities of (5) and (6) remained.

However, as the collision frequency increases significantly when the density approaches infinity, it is necessary to use another model where $\nu$ is a function of $n$. The density dependence of the collision frequency is usually given by $\nu(n)=2.9 \times 10^{-6} T^{-3 / 2} n \ln \Lambda$ in cgs-units. Here, $\ln \Lambda$ is the Coulomb logarithm, which has a rather complicated dependence on the cut-off angle for weak collisions, but for most practical applications ${ }^{15}$ can be put equal to 10 . In addition to a density dependent collision frequency, one may also consider the effect of a finite pressure in the momentum equation. However, it turns out that such a term will not stabilize divergent solutions of the type displayed in (5) and (6). In Ref. 16, the thermal influence on the nonlinear oscillations was studied for the constant collision frequency case. It was found that the shape of the periodic solutions can be fairly sensitive to a finite pressure, ${ }^{16}$ although the conditions for the divergences were essentially unaffected. By contrast, as will be demonstrated below, even a small collision frequency can be sufficient to remove the divergence, although only if that frequency is density dependent. Thus, we stress that the cold plasma approximation can still be useful when a density dependent collision frequency is considered.

For the remainder of this brief communication, we will focus on the linear dependence of $\nu$ on $n$. Hence, we shall represent $\nu$ as $\nu=\nu_{0} n / n_{0}$, where the constant $\nu_{0}$ is the collision frequency for $n=n_{0}$. With these preliminaries, we can improve the collisionless case by again looking for solutions where $N$ and $V$ depend only on time. Using (1) to express $V$ in terms of $n$, we then rewrite Eq. (4) as 

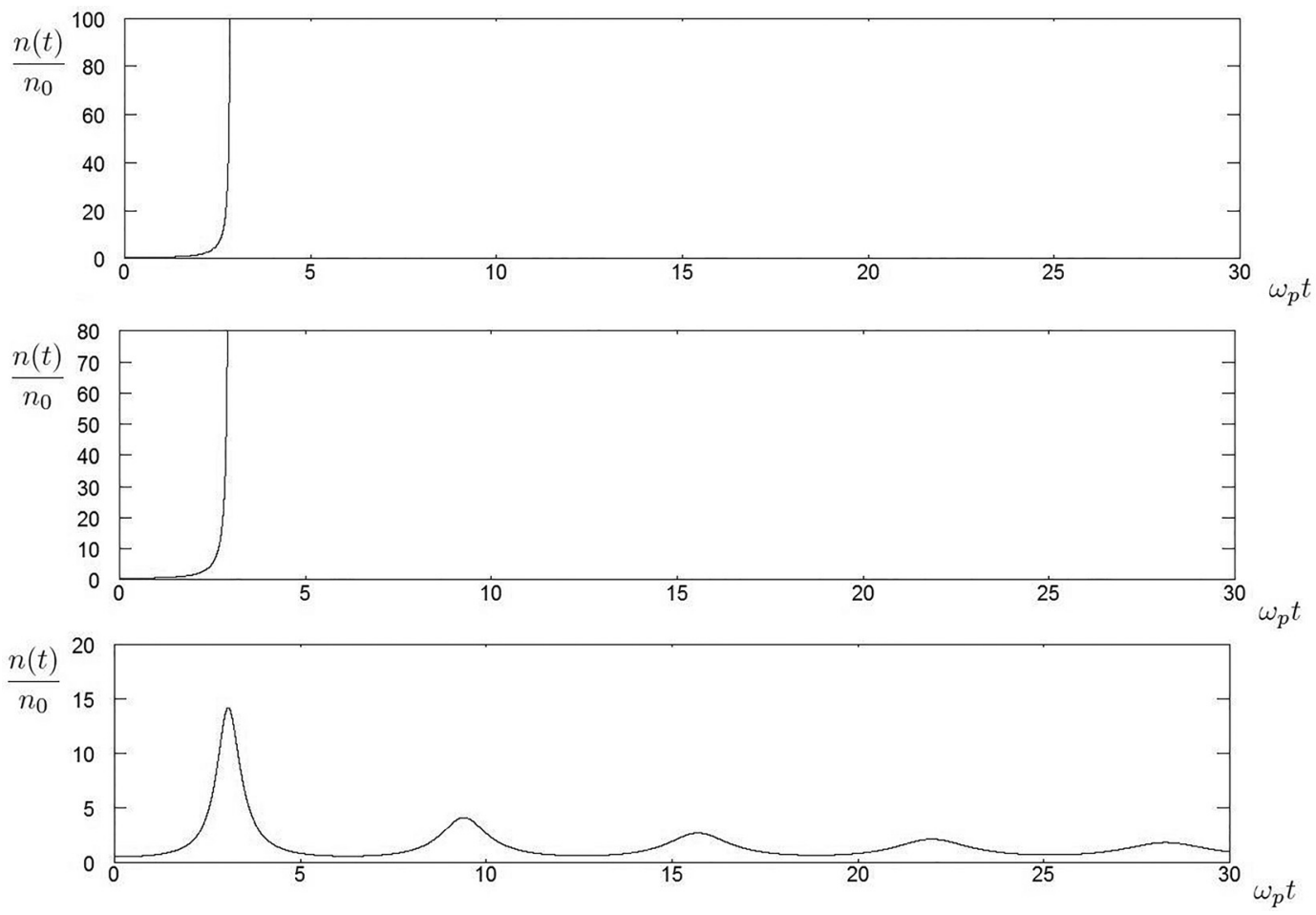

FIG. 1. The normalized density $n(t) / n_{0}$ plotted as a function of $\omega_{p} t$ for three different models. In all panels, we have used the same initial conditions, namely $n(t=0) / n_{0}=0.49$ and $d n(t=0) / d t=0$. The upper panel concerns the collisionless case $\nu_{0} / \omega_{p}=0$. In the middle panel, we have $\nu_{0} / \omega_{p}=0.05$, but the density dependence of $\nu$ is ignored. Finally, in the lower panel, the solution is based on the full model with a density dependent collision frequency and $\nu_{0} / \omega_{p}=0.05$

$$
\frac{d^{2}}{d t^{2}}\left(\frac{n_{0}}{n}\right)=-\omega_{p}^{2} \frac{\left(n_{0}-n\right)}{n}+\frac{\nu_{0}}{n} \frac{d n}{d t} .
$$

Equation (7) can easily be solved numerically. Our main purpose is to demonstrate that a finite collision frequency removes the singularities present in (5) and (6), but only if the density dependence is included. Thus, we compare three cases. Firstly, we let $\nu_{0}=0$ in Eq. (7). Secondly, we solve Eq. (7) with a finite collision frequency, but ignoring the density dependence [i.e., letting $\nu_{0} / n \rightarrow \nu_{0} / n_{0}$ in (7)]. Finally, Eq. (7) is solved with the full density dependence of $\nu$ as it stands. In Fig. 1, we thus plot three versions of the temporal evolution of $n$ for the same initial conditions using these three different models. As can be seen in both the upper and middle panels, the density diverges after a finite time. The middle panel is almost identical to the upper panel. The only effect of the constant collision frequency is thus to marginally adjust the time of divergence. By contrast, in the lower panel (with the density dependent collision frequency), we see a nonlinear oscillation with a damping that is initially strong, but then reduces when the amplitude of the oscillation is diminished. The removal of the divergence clearly demonstrates the necessity to include a finite, although very small, collision frequency, in order to significantly improve the collisionless case.

Similar effects occur due to ionization and attachment phenomena. In that case, we replace the right hand side in the continuity equation with a term $\gamma$ which is a function of the magnitude of the electric field. ${ }^{17}$ Using Eq. (3), we then

instead consider a simple model where $\gamma=\gamma(n)$. The solution will then turn out to be analogous to that where a model collision frequency $\nu(n)$ has been included. Surface effects can also play a similar role (e.g., Refs. 13 and 18-20) and lead to an equation related to Eq. (7).

${ }^{1}$ R. Z. Sagdeev and A. A. Galeev, Lectures on the Non-Linear Theory of Plasma, IC/66/64 (International Centre for Theoretical Physics, Trieste, Italy, 1964).

${ }^{2}$ R. C. Davidson, Methods in Nonlinear Plasma Theory (Academic Press, London, 1972).

${ }^{3}$ P. K. Shukla, "Nonlinear plasma science," Phys. Scr. T 82, 4 (1999),

${ }^{4}$ G. Brodin and L. Stenflo, Phys. Lett. A 378, 1632 (2014).

${ }^{5}$ B. Sahu, A. Sinha, and R. Roychoudhury, Phys. Plasmas 22, 092306 (2015).

${ }^{6}$ M. Karmakar, C. Maity, and N. Chakrabarti, Phys. Plasmas 23, 064503 (2016).

${ }^{7}$ D. A. Diver and E. W. Laing, Phys. Plasmas 23, 122103 (2016).

${ }^{8}$ P. S. Verma, Phys. Plasmas 23, 122125 (2016).

${ }^{9}$ H.-F. Liu and L.-M. Zhu, Phys. Plasmas 24, 034503 (2017).

${ }^{10}$ S. Pramanik and C. Maity, Phys. Plasmas 24, 084504 (2017).

${ }^{11}$ M. Akbari-Moghanjoughi, Phys. Plasmas 24, 092117 (2017).

${ }^{12}$ G. Brodin and L. Stenflo, Phys. Lett. A 381, 1033 (2017).

${ }^{13}$ O. M. Gradov, Phys. Lett. A 381, 3893 (2017).

${ }^{14}$ P. S. Verma, Phys. Lett. A 381, 4005 (2017).

${ }^{15}$ F. F. Chen, Introduction to Plasma Physics and Controlled Fusion, 3rd ed. (Springer, Heidelberg, 2016).

${ }^{16}$ L. Stenflo and G. Brodin, Phys. Plasmas 23, 074501 (2016).

${ }^{17}$ D. Anderson, A. V. Kim, M. Lisak, V. A. Mironov, A. M. Sergeev, and L. Stenflo, Phys. Rev. E 52, 4564 (1995).

${ }^{18}$ M. Y. Yu, Phys. Rev. A 28, 1855 (1983).

${ }^{19}$ A. R. Karimov, J. Plasma Phys. 75, 817 (2009).

${ }^{20}$ M.-J. Lee and Y.-D. Jung, Phys. Plasmas 22, 022125 (2015). 